\title{
Indian Classical Music Therapy - an effective therapeutic tool.
}

\author{
Rajam Shanker \\ Sangeetha Alankara-Carnatic Classical \\ $M$ A Vedic Astrology \\ Indian Classical Music Therapist \\ www.rajamsmusictherapy.com
}

Sounds, scales, variable pitches and frequencies, all of these are being employed by the scientific community to reach out, communicate and evoke a response from life forms in the Outer Universe. The Human Body is nothing but an Inner Universe which responds to physical and neural communication. Music, deployed in a calibrated dosage evokes a neural response. For the therapist, music as sound is a means to an end - an instrument for creating the sensation of hearing, a transmission of controlled energy that is perceived by the ear, processed by the brain and resonates in the energy centres of the body as a symbol of movement.

Musical notes are predominantly therapeutic. An individual or group can consciously exercise a choice of playing or listening to a selected Swara (Note), Raga (Scale), or composition and interpret their own physical and emotional response to the same. This generalization does not always hold true when they are employed as therapeutic intervention tools, where substantially, it is face-to-face, one-to-one. Clients could be verbal or nonverbal, communicative or otherwise, individuals or groups, participation could be active or passive - the choice of techniques employed by the therapist will be determined by these variables. Preliminary evaluation helps determine what caused or triggered the disability and / or current state of distress. You deal with the root causes, not the symptoms. Every individual's need and response to Music Therapy is unique. One cannot specify the duration required by the client to practice / be treated. The progress stages too vary depending upon individual degree of ability. For normal individuals however, like Yoga, the use of music to heal, improve and maintain wellness can be a lifelong practice.

WFMT - World Federation of Music Therapy has enunciated that cultural, social and political contexts be factored into choice of the genre of music the client or group is comfortable with. Carnatic, Hindustani, Indigenous, Folk, Western Classical are all choices available to the Indian Music Therapist in consultation with the individual or group clientele. Appropriate scale, note, pitch and beat form the component ingredients of Music as a therapeutic tool. 


\section{Music and its relationship with Energy Centres in the Human Body}

Indian Carnatic Classical Music, its infinite application variables and importantly, its spiritual connect are used by the Music Therapist as a means of establishing communication with the human body's main and subsidiary energy centers; it's extremely precise and elaborate structure of ragas (scales), swara (notes), srutis (pitch) and talas (beats) largely facilitates the requirement of specific design and calibrated delivery of music as deployed in therapeutic intervention. Frequency specific swara can be generated and delivered by the therapist.

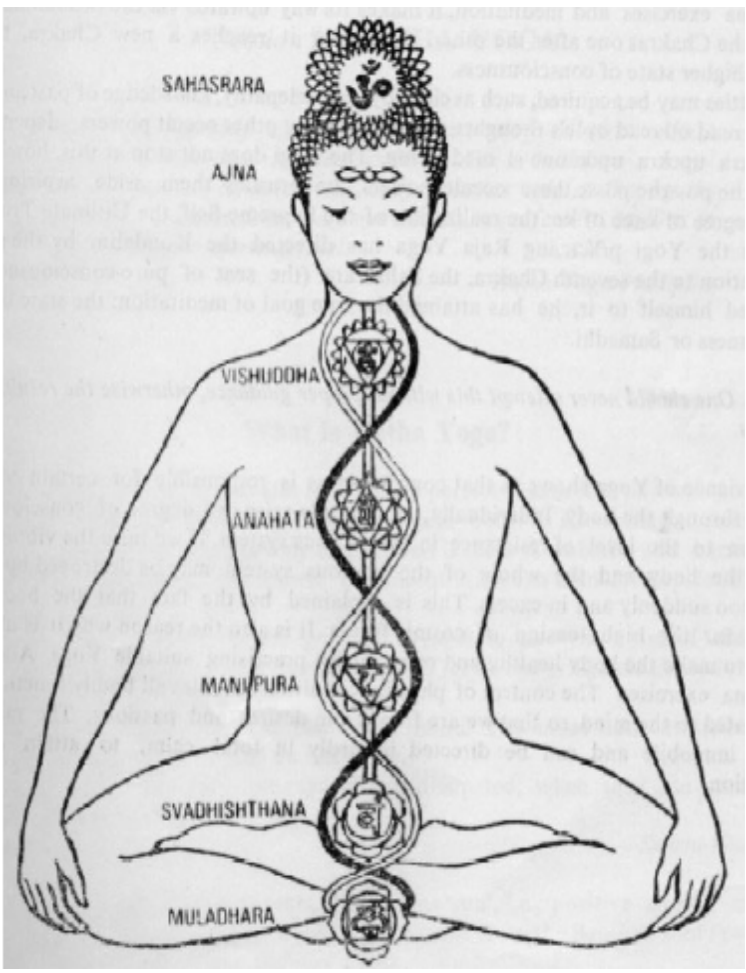

Image.1 Chakras - Energy Centres In the Human Body

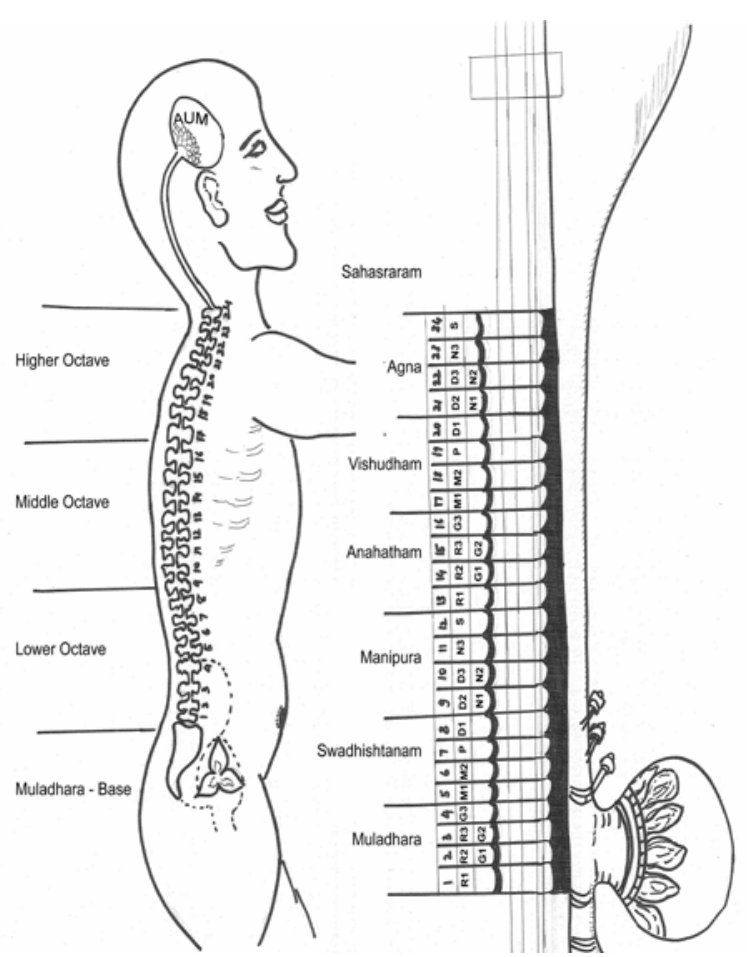

Image.2 Courtesy - Sangeetakalpadrumam

Look at the Image. 1 on the left. 7 Chakras [Energy Centers], 7 Swaras [Notes], 72 Melakarta Ragas [Scales] in Carnatic Classical Music and 72 Thousand Naadis [ Energy Channels ] - Music Therapy establishes a relationship between them. Shown are the seven main Chakras or interconnected energy centres of the human body; individually they exercise control over a specific group of internal organs and the body's functional mechanism and each has a rhythmic pulsation or vibration. Image 2 is a Human Body - Veena interface. It is significant that many Indian Classical Music String Instruments have the same structural interface, establishing a relationship between their generation of a musical note and its resonating response in the human body. 
Indian Classical Music, and indeed music universally, is built on the foundation of the basic seven notes or swaras - sa, re, ga, ma, pa, da, ni. $\quad \boldsymbol{S a}$ is the base note and foundation on which whole edifice of music is built. Similarly, Muladhara Chakra is the base Chakra. Consequently, with this at the base and the Sahasrara Chakra at the apex and represented by $\mathbf{N i}$ the human body's rhythmic balance and functional harmony is maintained. When there is a deficiency or weakening in a particular chakra or energy center, the functioning of the organs under its control display a disability or unhealthy performance. The swara associated with the particular chakra is specifically calibrated in its delivery by the therapist, and employed to provide the corrective charge to boost this energy center. Again, the importance of calibrated delivery of music cannot but be emphasized. The practice and response is case specific particularly when it is employed as therapeutic intervention

Indian Classical Music employed as an instrument of Music Therapy is unique in its pursuit and practice - drawing notes from deep within and use it to pulsate and vibrate a targeted chakra. The emphasis again is on calibration to harmonize and raise the level of consciousness. If the vibrations or resonant pulsations arriving and passing through the body are in excess or arrive too quickly, they could set off a higher level of resistance, and the results could be counterproductive. Properly practiced, results are positive and sustained.

Indian Classical Music Therapy is inviting increasing acceptance in the conservative Indian environment because of its greater social acceptability - it has always been part of our social milieu and traditions. There is still substantial social reluctance to admitting to emotional and neurological disabilities and seeking possibly invasive medical intervention

The advantage of multi-disciplinary collaboration is that it provides for systematic evaluation and clinical validation of visible and perceptible changes observed. Each Client's degree and extent of disabilities is distinctly individual which a detailed preliminary evaluation of a number of inputs establishes. Consequently, the Music Therapy protocol deployed is client specific, calibrated, monitored, modified and continuously evaluated. Therapeutic intervention is, I reiterate, calibrated and client specific - there are no one-size-fits-all and over-the-counter remedies. To distinguish between employing and benefitting from Music - knowledge of Music and its infinite variables is, in my opinion, a prerequisite for one to develop, practice and employ 
Music Therapy. To benefit from it one needs to have both, a receptive frame of mind and positive attitude for music therapy to be effective. This also holds true for both, wellness and therapeutic interventions.

\section{RAGAS FOR RELIEF!}

Indian Classical Music and its spectrum of Ragas, Swaras, Sruti and Talas and the option to improvise and extemporize provides infinite variables to a Music Therapist required to initiate a client specific intervention. [ Over-the-counter self-selected options are however inevitable] .

\section{MELAKARTA RAGAS - THE INDIAN CLASSICAL MUSIC THERAPIST'S PHARMACOEPIA}

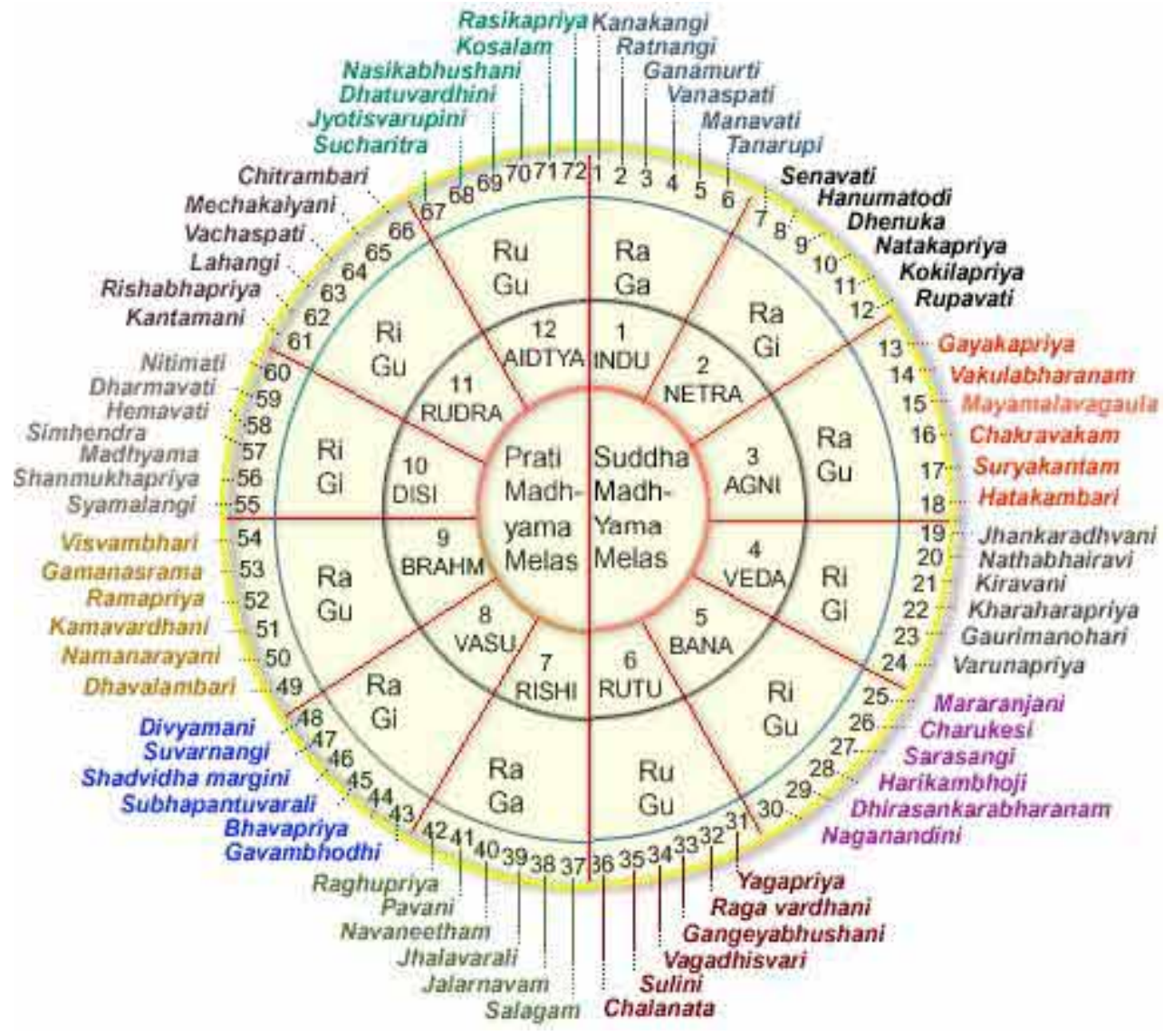

Multiple renderings and compositions in all the above ragas, each invested with differing levels of Bhava [emotion] are commercially available. The emphasis is on consciously selecting one appropriate to your 'musical finger-print'

\section{Rajam Shanker}




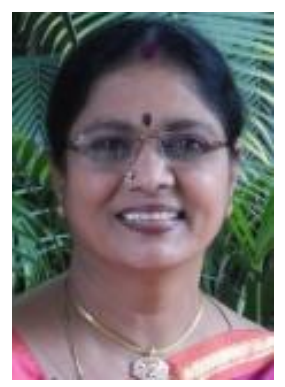

Rajam Shanker, born 1952, is a graduate in Carnatic Classical Music from the Telugu University, Hyderabad and Sangeeta Alankara with distinction from Akhil Bharatiya Gandharva Mahavidyalaya, the National Music University. She was initiated into the practice of Indian Traditional Music Therapy by her mentor and Guru, Sangeetha Mahamahopadhyaya Sri Kollegal R Subramanyam, the eminent Vaggeyakara, lyricist, musician and grammarian. She also has a Masters in Vedic Astrology and researches its employment as a medical evaluation and diagnostic tool.

Excerpts can be viewed on her website: www.rajamsmusictherapy.com 